\title{
The sensitivity of stratocumulus-capped mixed layers to cloud droplet concentration: do LES and mixed-layer models agree?
}

\author{
J. Uchida ${ }^{1}$, C. S. Bretherton ${ }^{2}$, and P. N. Blossey ${ }^{2}$ \\ ${ }^{1}$ Department of Applied Mathematics, University of Washington, Seattle, Washington, USA \\ ${ }^{2}$ Department of Atmospheric Science, University of Washington, Seattle, Washington, USA
}

Received: 5 November 2009 - Published in Atmos. Chem. Phys. Discuss.: 2 December 2009

Revised: 16 April 2010 - Accepted: 17 April 2010 - Published: 3 May 2010

\begin{abstract}
The sensitivity of a stratocumulus-capped mixed layer to a change in cloud droplet concentration is evaluated with a large-eddy simulation (LES) and a mixed layer model (MLM). The strength of the second aerosol indirect effect simulated by the two model types agrees within $50 \%$ for cases in which the LES-simulated boundary layer remains well mixed, if the MLM entrainment closure includes the effects of cloud droplet sedimentation.

To achieve this agreement, parameters in the MLM entrainment closure and the drizzle parameterization must be retuned to match the LES. This is because the LES advection scheme and microphysical parameterization significantly bias the entrainment rate and precipitation profile compared to observational best guesses. Before this modification, the MLM simulates more liquid water path and much more drizzle at a given droplet concentration than the LES and is more sensitive to droplet concentration, even undergoing a drizzle-induced boundary layer collapse at low droplet concentrations. After this modification, both models predict a comparable decrease of cloud liquid water path as droplet concentration increases, cancelling 30-50\% of the Twomey effect for our case. The agreement breaks down at the lowest simulated droplet concentrations, for which the boundary layer in the LES is not well mixed.

Our results highlight issues with both types of model. Potential LES biases due to inadequate resolution, subgrid mixing and parameterized microphysics must be carefully considered when trying to make a quantitative inference of the second indirect effect from an LES of a stratocumulustopped boundary layer. On the other hand, even slight internal decoupling of the boundary layer invalidates the central
\end{abstract}

Correspondence to: C. S. Bretherton (breth@washington.edu) assumption of an MLM, substantially limiting the range of conditions that MLM-predicted sensitivities to droplet concentration are meaningful.

\section{Introduction}

The indirect effect of anthropogenic aerosol on clouds and thereby on the global radiation balance remains a key uncertainty in climate modeling and prediction. In particular, the cloud droplet number, cloud fraction and liquid water path (LWP) of subtropical marine stratocumulus (Sc) cloud decks respond to changes in aerosol in ways global models struggle to represent, because they involve a subtle interplay between turbulence, vertical structure, entrainment and evaporating precipitation. Low aerosol concentrations often lead to marine boundary layers with reduced cloud fraction, more precipitation and a more decoupled and cumuliform character, as seen both from observations of pockets of open cells (Comstock et al., 2005; Stevens et al., 2005a; Petters et al., 2006; Wood et al., 2008) and large-eddy simulations or LES (Stevens et al., 1998; Ackerman et al., 2003; Xue et al., 2008; Savic-Jovcic and Stevens, 2008). Intermediate aerosol concentrations can support fully cloud-covered but drizzling boundary layers in which subcloud cooling from evaporating drizzle inhibits turbulent mixing and reduces entrainment of dry air, supporting stratocumulus with a high liquid water path, e.g. Lu and Seinfeld (2005). High aerosol concentrations produce small cloud droplets that do not sediment out of the entrainment zone, promoting mixing-induced evaporative cooling that enhances cloud top entrainment and can thin the Sc layer (Ackerman et al., 2004; Bretherton et al., 2007). Thus, ship or volcano tracks of enhanced aerosol concentration and reduced droplet effective radius in nonprecipitating Sc often appear to have lower LWP than the surrounding

Published by Copernicus Publications on behalf of the European Geosciences Union. 
cloud (Coakley and Walsh, 2002; Gasso, 2008); though there are issues of interpretation (Ackerman et al., 2003).

Models are required to interpret and generalize these limited observations. Two types of models that have been widely used are LES and mixed-layer models (MLMs). LES models realistically represent the interaction of turbulence, cloud processes and radiation within the constraints of grid resolution. However, they are computationally complex. Model intercomparisons (Stevens et al., 2005b; Ackerman et al., 2009) show that LES as a group cannot reliably simulate entrainment into Sc through the sharp capping inversion, often leading to simulated Sc layers with too small LWP. Furthermore, LES microphysical parameterizations for predicting precipitation development have numerous uncertainties (Ackerman et al., 2009).

MLMs have been used for several studies of Sc sensitivity to aerosols in which long-term behavior and conceptual simplicity have been emphasized (Baker and Charlson, 1990; Pincus and Baker, 1994; Wood, 2007; Caldwell and Bretherton, 2009). MLMs break down when the boundary layer becomes decoupled, but Sc-capped boundary layers are sometimes well-mixed even when droplet concentrations are low and they start to lightly drizzle, e.g. the SE Pacific EPIC observations of Bretherton et al. (2004). MLMs usually use empirical observationally-informed parameterizations of entrainment and cloud microphysical processes in terms of the bulk variables that MLMs predict (e.g. mean cloud base and inversion height). An MLM provides a simpler and complementary view to LES of Sc-capped mixed layers, albeit one with restricted applicability.

The goal of this paper is to test whether LES and mixed layer models respond similarly to changes in assumed cloud droplet concentration in Sc-capped mixed layers, in which both model types might be expected to apply. We view this as a proxy for their response to aerosol perturbations. To this end, we compare sets of identically forced and initialized nocturnal LES and MLM simulations run out 5 days, focusing on the evolution of LWP.

Our study is similar to that of Sandu et al. (2009), hereafter S2009. It was conceived independently and in parallel with S2009. They compared 72-h LES and MLM simulations of polluted and pristine cloud-topped boundary layers, using forcings idealized from a model intercomparison of the diurnal cycle of Sc in the California coastal zone. Our setup is somewhat simpler (no diurnal cycle) and more idealized. S2009 found that an increase in droplet concentration decreased LWP in their LES, but increased LWP in their MLM. They concluded that an MLM is not a useful tool for looking at cloud-aerosol interactions even for relatively well-mixed boundary layers. We reach the more optimistic conclusion that for Sc-capped mixed layers, the sensitivity of LWP to cloud droplet concentration is comparable (within better than a factor of two) for our LES and an appropriately configured MLM. In the discussion section, we will trace this difference in conclusions between the studies mainly to the choice of
MLM entrainment parameterization. In particular, we argue that it is vital to consistently build into the MLM the response of entrainment to cloud droplet sedimentation and drizzle.

\section{Models and simulation setup}

Our simulations are based on the GEWEX Cloud System Study (GCSS) nocturnal nonprecipitating Sc case specifications for single column models (Zhu et al., 2005). This case was idealized from Research Flight 1 (RF01) of the Second Dynamics and Chemistry of Marine Stratocumulus Experiment, DYCOMS-II (Stevens et al., 2003). This case featured a well mixed Sc-capped mixed layer in which MLM and LES can reasonably be compared with each other and with observations. Salient details and changes to the case specifications are discussed below.

\subsection{LES}

The LES used in this study is version 6.7 of the System for Atmospheric Modeling (SAM), kindly supplied by Marat Khairoutdinov and documented by Khairoutdinov and Randall (2003). A Deardorff sub-grid turbulent diffusivity with prognostic subgrid TKE is used. A two-category singlemoment bulk microphysics parameterization is used in which water is partitioned into a vapor mixing ratio $q_{v}$, a cloud liquid water mixing ratio $q_{c}$ and a rain water mixing ratio $q_{r}$. The monotonicity-preserving scheme of Smolarkiewicz and Grabowski (1990) is used for advecting three thermodynamic scalars, the total water mixing ratio $q_{t}=q_{v}+q_{c}$, the rain water mixing ratio, and the liquid static energy $s_{l}=c_{p} T+g z-L q_{c}$ (where $z$ is height, $c_{p}$ is the specific heat of dry air at constant pressure, $g$ is gravity, and $L$ is the latent heat of vaporization). The cloud liquid water and temperature are diagnosed from these scalars using the assumption of exact grid-scale saturation in cloudy grid cells. The Khairoutdinov and Kogan (2000) scheme is used for conversion between cloud and rain water. Cloud droplet sedimentation is included following Eq. (7) of Ackerman et al. (2009), based on a log-normal droplet size distribution with a geometric standard deviation $\sigma_{g}=1.2$.

The LES neglects the finite timescales both for mixing of cloudy and subsaturated air within a grid cell, and for the evaporation of cloud droplets in subsaturated air. Both these effects would decrease the efficiency of cloud-top entrainment in the LES, because they slow down the evaporative cooling of air undergoing turbulent mixing in the entrainment zone (Hill et al., 2009); the finite evaporation timescale is sensitive to droplet size and would act to enhance the feedback of sedimentation on entrainment simulated by our LES (to which our MLM is also tuned).

Following the GCSS case specifications, we used an idealized radiative heating parameterization, as further discussed below. 
All simulations use a uniform $25 \mathrm{~m}$ horizontal and $5 \mathrm{~m}$ vertical grid spacing over a $2.4 \times 2.4 \times 1.5 \mathrm{~km}$ domain with doubly-periodic boundary conditions, and an overlying sponge layer in which the vertical grid spacing rapidly coarsens.

\subsection{MLM}

The MLM, described by Bretherton and Wyant (1997) and Caldwell and Bretherton (2009), predicts the mixed layer moist static energy $h=c_{p} T+g z+L q_{v}$, total (vapor plus cloud liquid) water mixing ratio $q_{t}$ and inversion height $z_{i}$. It allows for continuously varying profiles of radiative heating and precipitation flux within the mixed layer. The precipitation flux includes sedimentation and drizzle components following Caldwell and Bretherton (2009). The sedimentation flux is related to the cloud liquid water content exactly as in the LES. The drizzle flux combines an observationallyderived estimate of cloud base drizzle (Comstock et al., 2004) as proportional to LWP/ $N^{1.75}$ with a simple representation of subcloud drizzle evaporation.

The MLM entrainment parameterization is based on Nicholls and Turton (1986):

$w_{e}=A w_{*}^{3} /\left(z_{i} \Delta b\right)$.

where $w_{e}$ is the entrainment rate, $w_{*}$ is a convective velocity computed as the cube root of 2.5 times the vertical integral of the buoyancy flux, $\Delta b$ is the inversion jump (denoted by $\Delta$ ) of virtual potential temperature expressed in buoyancy units, and $A$ is a nondimensional entrainment efficiency, assumed to have the form

$A=a_{1}\left\{1+a_{2} \chi_{*}\left(1-\Delta b_{s} / \Delta b\right) \exp \left(-a_{\text {sed }} w_{\text {sed }} / w_{*}\right)\right\}$.

Here, $a_{1}=0.2$ is the entrainment efficiency of a dry convective boundary layer, $\chi_{*}$ is the mixing fraction of overlying air needed to evaporate all the water out of the mixed layer air at the cloud top, and $\chi_{*} \Delta b_{s}$ is the resulting buoyancy change to the cloudy mixed layer air; it may be positive or negative ("buoyancy reversal") depending on the sign of the inversion jump of saturated buoyancy $\Delta b_{s}=g\left(\beta \Delta_{i} h / h-\Delta_{i} q_{t}\right)$ introduced in Appendix B of Grenier and Bretherton (2001). The thermodynamic coefficient $\beta$ (Randall, 1980)) is a weak function of pressure and temperature that is typically near 0.5 . The evaporative enhancement coefficient $a_{2}=25$ was empirically chosen following Caldwell and Bretherton (2009), using observations of entrainment and turbulent fluxes from DYCOMS-II cases RF01 (Stevens et al., 2005b) and RF02 (Ackerman et al., 2009) and estimates inferred from the diurnal cycle of Sc during EPIC 2001 (Caldwell et al., 2005).

Following Bretherton et al. (2007), the evaporative enhancement is reduced by an exponential factor dependent on an LES-tuned sedimentation feedback parameter $a_{\text {sed }}=9$ multiplied by the ratio of the mean droplet sedimentation speed $w_{\text {sed }}$ near cloud top to the convective velocity $w_{*}$. The sedimentation speed is computed from the quadratic dependence of the terminal velocity of small droplets to their radius, integrated across the assumed log-normal droplet size distribution. If the cloud-top liquid water content is $q_{\mathrm{li}}$, the air density is $\rho_{a}$, and the density of liquid water is $\rho_{w}$, we can define a volume-mean droplet radius $r_{i}=\left(3 \rho_{a} q_{\mathrm{li}} / 4 \pi \rho_{w} N\right)^{1 / 3}$ and

$w_{\text {sed }}=c r_{i}^{2} \exp \left(5 \log ^{2} \sigma_{g}\right)$,

where $c=1.19 \times 10^{8} \mathrm{~m}^{-1} \mathrm{~s}^{-1}$; we choose $\sigma_{g}=1.2$ as in the LES.

In an MLM, the liquid water profile is adiabatic and linearly increasing with height, and $q_{\mathrm{li}}$ is proportional to LWP $^{1 / 2}$. With typical values for all coefficients in Eq. (3),

$w_{\text {sed }} \approx 6.0 \times 10^{5} \mathrm{~m}^{-1} \mathrm{~s}^{-1} \cdot\left(q_{\mathrm{li}} / N\right)^{2 / 3}$

For comparison of LES results with the MLM entrainment closure (which involves $w_{\text {sed }}$ ), it is useful to diagnose an effective LES cloud-top droplet sedimentation velocity. The LES has a somewhat rounded vertical profile of $q_{c}$ near the inversion, rather than the sharp-topped triangular vertical profile of $q_{c}$ of the MLM, so it is ambiguous how to do this. Our approach is to calculate for the LES an equivalent adiabatic cloud-top liquid water content $q_{\mathrm{li}} \propto \mathrm{LWP}^{1 / 2}$ that corresponds to the given value of LWP. Substituting this into Eq. (4) gives

$w_{\text {sed }} \approx 9.1 \times 10^{3} \mathrm{~kg}^{-1 / 3} \mathrm{~m}^{-1 / 3} \mathrm{~s}^{-1} \cdot\left(\mathrm{LWP} / N^{2}\right)^{1 / 3}$,

The self-consistency of the MLM simulations is tested by computing a decoupling indicator, the buoyancy integral ratio or BIR (Bretherton and Wyant, 1997). The BIR is defined as the vertical integral of the negative buoyancy flux in subcloud layer to the vertical integral of the positive buoyancy flux over the rest of the mixed layer. A BIR exceeding 0.10.2 suggests that the boundary layer will not remain wellmixed and the MLM is no longer appropriate (Bretherton and Wyant, 1997; Stevens, 2000).

\subsection{Radiative heating parameterization}

The RF01 case specification includes an idealized radiative heating parameterization used by both the LES and MLM. The net upwelling radiative flux at each height in a grid column is specified based on the column liquid water path above and below that height. This specification does not include the influence of droplet number on the radiative heating profile through its effect on the droplet effective radius. Larson and Kotenberg (2007) have described how one could account for this influence, which may affect entrainment rate by modulating how much radiative cooling occurs within the entrainment zone and by affecting the relationship between LWP and cloud emissivity, which is important for thin clouds.

The original specification produces no radiative cooling in clear air columns, which unrealistically ignores the emissivity of water vapor. Since some of our LES runs produced 
only partial cloud cover, we enforced a minimum average cooling rate of $2 \mathrm{~K} \mathrm{day}^{-1}$ between the sea-surface and the inversion in each grid column. If the simulated cloud in the column does not produce at least this average cooling (this requires a column LWP of about $10 \mathrm{~g} \mathrm{~m}^{-2}$ ), the average cooling is brought up to this threshold by adding a heightindependent increment of liquid water within the boundary layer for the radiative cooling profile calculation.

In the RF01 case specification, a strongly stratified layer with strong radiative cooling is specified just above the inversion. In an MLM, free tropospheric temperature and humidity profiles are specified all the way down to the inversion, and it is inconvenient to include a free-tropospheric layer with extra radiative cooling. Thus, we did not include the RF01-specified layer of enhanced radiative cooling and stratification above the inversion. Instead, we crudely compensated by increasing the inversion temperature jump $2 \mathrm{~K}$ from the RF01 specifications and using a linear temperature profile above that.

\subsection{Initial state, free-tropospheric profiles and surface fluxes}

Following the GCSS RF01 specifications, the simulations are initialized with a mixed layer with an inversion height $z_{i}=840 \mathrm{~m}$, a total water mixing ratio $q_{t}=9 \mathrm{~g} \mathrm{~kg}^{-1}$ and a moist static energy $h=317.03 \mathrm{~kJ} \mathrm{~kg}^{-1}$, producing a Sc cloud of initial thickness $250 \mathrm{~m}$. The large-scale subsidence varies linearly with height $z$ with a horizontal divergence $D=3.75 \times 10^{-6} \mathrm{~s}^{-1}$.

As in the RF01 specifications, we assume a heightindependent free-tropospheric humidity $q^{+}(z)=1.5 \mathrm{~g} \mathrm{~kg}^{-1}$. As motivated above, we use a linear free-tropospheric moist static energy profile

$h^{+}(z)=h^{+}(0)+\left(6 \mathrm{~kJ} \mathrm{~km}^{-1}\right) z$,

By choosing $h^{+}(0)=303.92 \mathrm{~kJ} \mathrm{~kg}^{-1}$, we obtain a roughly moist-adiabatic free-tropospheric temperature profile that is $2 \mathrm{~K}$ warmer than the RF01 specifications at the initial inversion height $z_{i}=840 \mathrm{~m}$. The above-inversion radiative cooling is specified to balance subsidence warming, and the humidity is height-independent, so the temperature and moisture profiles do not drift above the inversion.

Following the RF01 SCM case specification, we calculate surface heat and moisture fluxes using a sea-surface temperature (SST) of $292.5 \mathrm{~K}$. The LES wind profile is forced by the specified geostrophic winds, and the surface fluxes are computed using Monin-Obukhov theory and the simulated lowest-level wind speed. The MLM uses a bulk aerodynamic formula with the surface wind speed of $V=7.35 \mathrm{~m} \mathrm{~s}^{-1}$ specified in the SCM case description and a transfer coefficient $C_{T}=0.001 \mathrm{~V}$ for heat and moisture.

\section{Results}

First, we compare LES of the modified RF01 case described above with four different specified values of droplet concentration $N=150,50,30$ and $10 \mathrm{~cm}^{-3}$. The 5 day simulations, which we refer to as N150, N50, N30 and N10, are long enough to see the boundary layer depth and cloud thickness evolve much of the way toward an equilibrium.

Then, identically initialized and forced MLM simulations are compared with the LES results. We derive a modified MLM entrainment closure that better matches the LES results, which are affected by numerical over-entrainment. We compare the MLM-predicted response of the LWP to the droplet concentration to that of the LES, discarding cases for which the MLM internally predicts decoupling based on the criterion $\mathrm{BIR}>0.2$.

\subsection{LES results}

Figure 1 shows some domain-averaged statistics from the four LES cases. The first two hours, during the LES spinup, are not plotted; thereafter hourly-average values are shown. During the spinup, the LWP in all four cases quickly drops from the initial specification of $60 \mathrm{~g} \mathrm{~m}^{-2}$ to $30-35 \mathrm{~g} \mathrm{~m}^{-2}$. The statistics of the N150 case for the next few hours after this spinup can be compared with the RF01 observations, since the observed cloud droplet concentration was $140 \mathrm{~cm}^{-3}$. The simulated entrainment rate of around $4.5 \mathrm{~mm} \mathrm{~s}^{-1}$ only slightly exceeds the observed $4 \mathrm{~mm} \mathrm{~s}^{-1}$, but the simulated entrainment is maintained by a cloud whose LWP is much less than the observed LWP of $50-60 \mathrm{~g} \mathrm{~m}^{-2}$.

This manifestation of over-entrainment is an important systematic error of the LES for cloud-aerosol-precipitation interaction. Firstly, too thin a cloud will not precipitate as easily. Secondly, the total longwave cooling across a thin Sc layer is more sensitive to a given change in LWP than for a thick Sc layer (e.g. Fig. 7 of Stevens et al. (2005b)). This distorts the feedback between aerosols, LWP and the radiative driving of the boundary layer.

In general, the N30 and N50 cases behave similarly to the N150 case, with little or no cloud base drizzle and a cloud fraction exceeding 0.8. All three cases maintain a high entrainment rate that deepens the boundary layer and raises the minimum cloud base during the simulation.

Nevertheless, there are clear differences between these three cases. The N30 case has larger LWP and higher convective velocity than the N150 case throughout the simulations. The LWP difference develops within the first few hours, when N150 has a larger $w_{e}$ and more entrainment drying. In all three cases, the LWP and cloud fraction decrease during the first day (by which we mean a 24 hour time interval, not a period with sunlight). This (especially the reduced cloud fraction) decreases the boundary layer radiative cooling, acting as a negative feedback on the entrainment. Because it has the lowest cloud fraction and LWP, the 

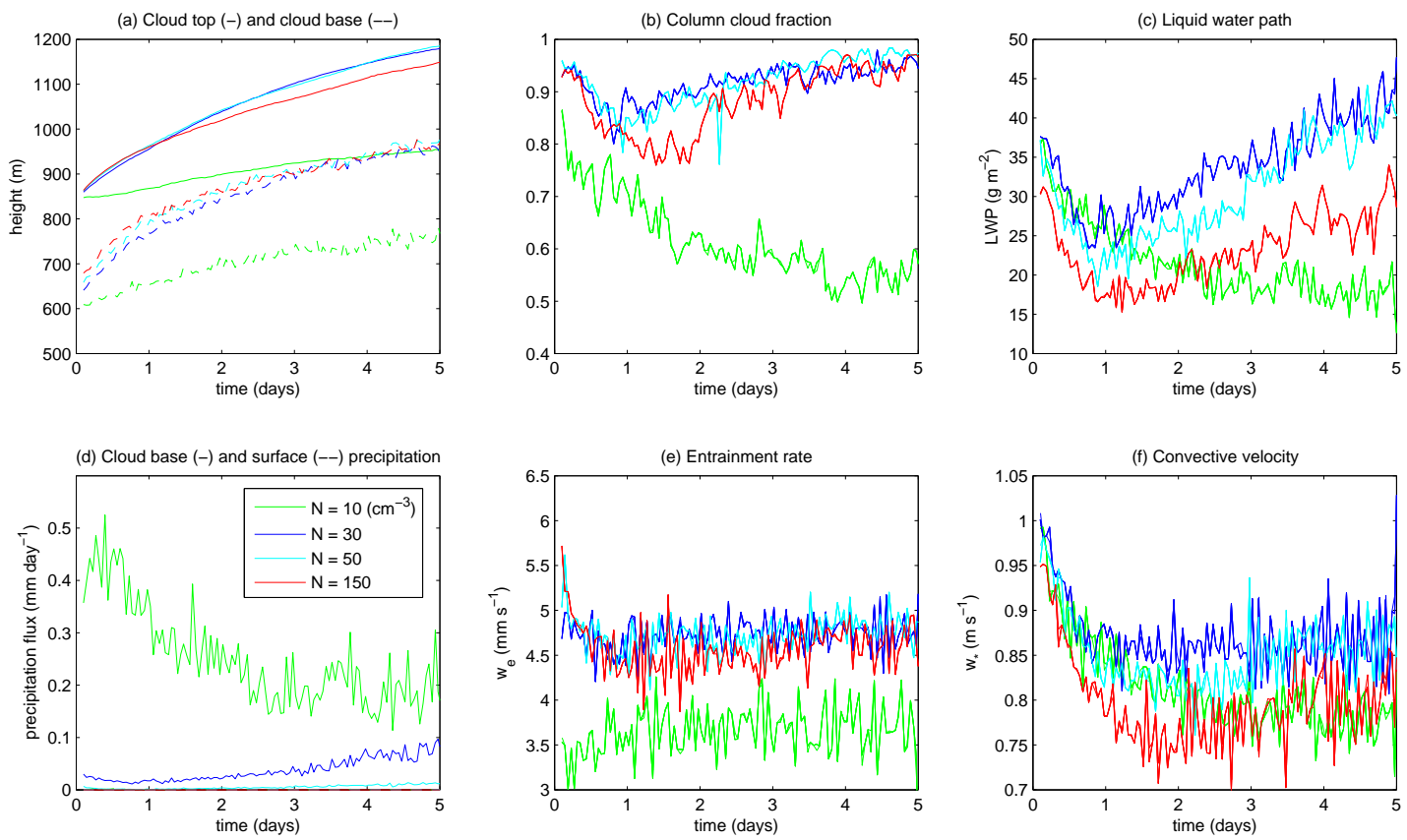

Fig. 1. LES time series for $N=10,30,50$ and $150 \mathrm{~cm}^{-3}$ : (a) highest cloud top and lowest cloud base, (b) column cloud fraction, (c) LWP, (d) Cloud base precipitation averaged across all grid columns - surface precipitation is negligible in all cases, (e) entrainment rate, (f) convective velocity.

N150 simulation has a total radiative flux divergence across the boundary layer which averages a few percent lower than for N50 and 30 during the simulation (not shown), and a correspondingly lower entrainment rate. Consequently, after a day the N150 inversion height becomes lower than for N30.

We attribute these trends to simulated droplet sedimentation effects on entrainment (Bretherton et al., 2007). For a given liquid water content, the mean cloud droplet size, and hence the sedimentation velocity, increases as $N$ decreases. More sedimentation decreases the efficiency of entrainment by removing the water droplets from the cloud top, so there is less water to evaporatively cool mixtures of entrained and boundary-layer air.

We quantify this argument by defining an LES entrainment efficiency by analogy with the MLM entrainment closure,

$A_{\mathrm{LES}}=w_{e} z_{i} \Delta b / w_{*}^{3}$.

There are only slight differences in the three cases between the evolution of $w_{e}, z_{i}$, and the buoyancy jump $\Delta b$ (measured between $25 \mathrm{~m}$ above and below $z_{i}$ ). However, the convective velocity $w_{*}$ (determined as the cube root of 2.5 times the vertical integral of the LES-derived buoyancy flux) drops to a 15 day average value about 7\% lower for N150 than for N30, with N50 lying in between. This is consistent with a roughly $20 \%$ larger entrainment efficiency for $\mathrm{N} 150\left(A_{\mathrm{LES}} \approx 1.2\right)$ than for $\mathrm{N} 30$ ( $\left.A_{\mathrm{LES}} \approx 1.0\right)$. The reduction in convective velocity is accomplished by decreased LWP, which causes cloud thinning and lower fractional cloud cover (less radiative driving), both of which diminish the vertically-integrated buoyancy production of turbulence. That is, the larger N150 entrainment efficiency does not lead to a larger sustained entrainment rate compared to N30. Instead, the N150 cloud layer entrains at about the same rate as N30, but accomplishes this entrainment with less turbulence and lower LWP.

In this light, we interpret the low LWP bias of the N150 simulation compared to observations as evidence that the LES is entraining too efficiently. As shown in the DYCOMS RF01 intercomparison, almost all LES models have this bias to varying degrees, and SAM's LWP is close to the median of the 16 participating LES models.

The N10 case is markedly different from the other three. It maintains a lower entrainment rate, a correspondingly lower inversion and cloud base height, and a lower cloud fraction. Initially, this case produces more than $0.2 \mathrm{~mm} \mathrm{~d}^{-1}$ cloud base drizzle; this reduces to $0.1 \mathrm{~mm} \mathrm{~d}^{-1}$ as its LWP drops below that of the other cases. The lower entrainment rate is mainly due to reduced cloud cover, which diminishes the radiative cooling of the boundary layer. The N10 cloud fraction is lower for a given LWP than for the other cases, perhaps because the subcloud drizzle-induced evaporative cooling is promoting horizontal inhomogeneity of the boundary layer.

Figure 2 shows selected profiles averaged over the period 2-2.25 days, using a normalized vertical coordinate $z / z_{i}$. This time was chosen to be long enough to allow all simulations to adjust into a phase in which their cloud properties are no longer rapidly evolving, yet short enough that they 

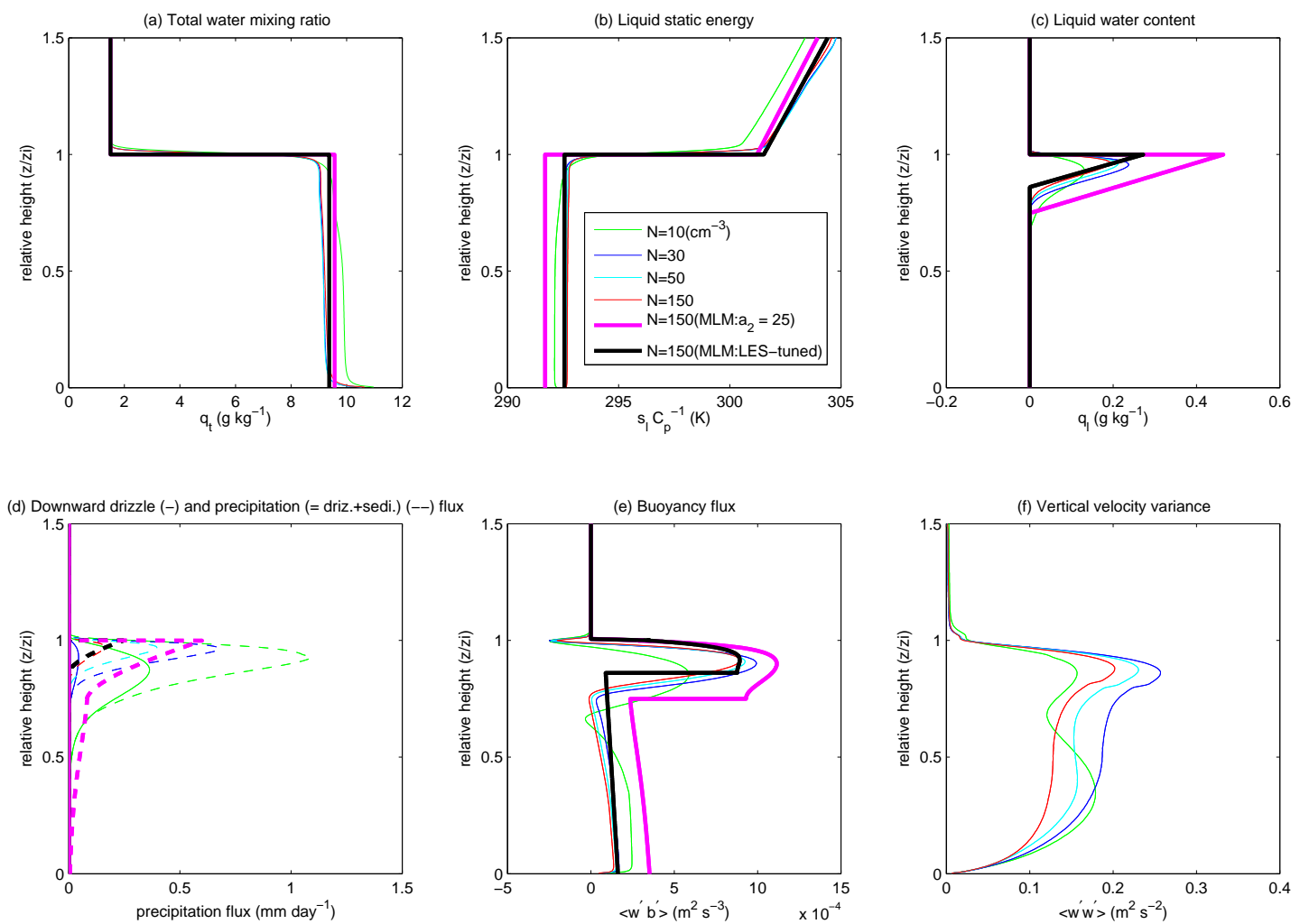

Fig. 2. LES profiles for $N=10,30,50$ and $150 \mathrm{~cm}^{-3}$, averaged from 2-2.25 days and plotted against an inversion-normalized vertical coordinate: (a) $q_{t}$, (b) $s_{l}$, (c) liquid water content, (d) downward drizzle and precipitation (=drizzle + sedimentation) fluxes, (e) buoyancy flux, (f) vertical velocity variance. In (a)-(e) the thick curves show MLM results for $N=150 \mathrm{~cm}^{-3}$ with the standard and LES-tuned entrainment closure for comparison.

all still have inversion heights less than $150 \mathrm{~m}$ apart, and hence comparable above-inversion conditions and inversion jumps. For N30, N50 and N150, the profiles of the moistadiabatically conserved variables, liquid static energy and total water mixing ratio, are nearly identical and vertically well mixed within the boundary layer. Thus, these cases are reasonable comparisons for an MLM. All three simulations produce a minimal drizzle flux with a maximum within the cloud layer of less than $0.1 \mathrm{~mm} \mathrm{~d}^{-1}$. The N30 case shows a much stronger sedimentation flux out of the cloud top than the N150 case. Due to the lower entrainment efficiency, the N30 cloud is slightly thicker and the buoyancy flux and vertical velocity variance are slightly higher than for N150. The N50 profiles closely resemble those for N30. The N10 case has much more drizzle, a kink in the cloud fraction profile indicating more variability in cloud base, vertical gradients in the conserved variable profiles in the upper part of the boundary layer, a level near cloud base at which buoyancy flux drops below zero, and a double-peaked vertical velocity variance. These are all indicators of a less well mixed boundary layer that an MLM cannot be expected to represent well.

\subsection{MLM results with standard entrainment closure}

Figure 3 shows MLM simulations for the N30, N50, and N150 cases forced identically to the LES runs shown in Fig. 1. For N150, the inversion deepens in the MLM simulation, but less rapidly than for the LES. The MLM maintains roughly double the LWP of the LES throughout the simulation. Its initial LWP is more consistent with the RF01 observational estimate of $60 \mathrm{~g} \mathrm{~m}^{-2}$ than is the LES, because as noted in Section 2.2, the MLM entrainment closure was tuned to match the observed entrainment efficiency for this case. The MLM buoyancy flux profile (magenta line in Fig. 2e) is qualitatively similar to the LES (red line), but with more buoyancy flux in the cloud layer and a larger convective velocity $w_{*}$. Because the MLM has a lower entrainment efficiency than the LES, its entrainment rate is smaller despite its larger $w_{*}$.

The N50 MLM simulation generates enough evaporating cloud base drizzle to significantly reduce buoyancy production of turbulence and entrainment. Thus, initially the boundary layer moistens, the cloud thickens and LWP increases faster than for N150, though ultimately a steady state is approached. 

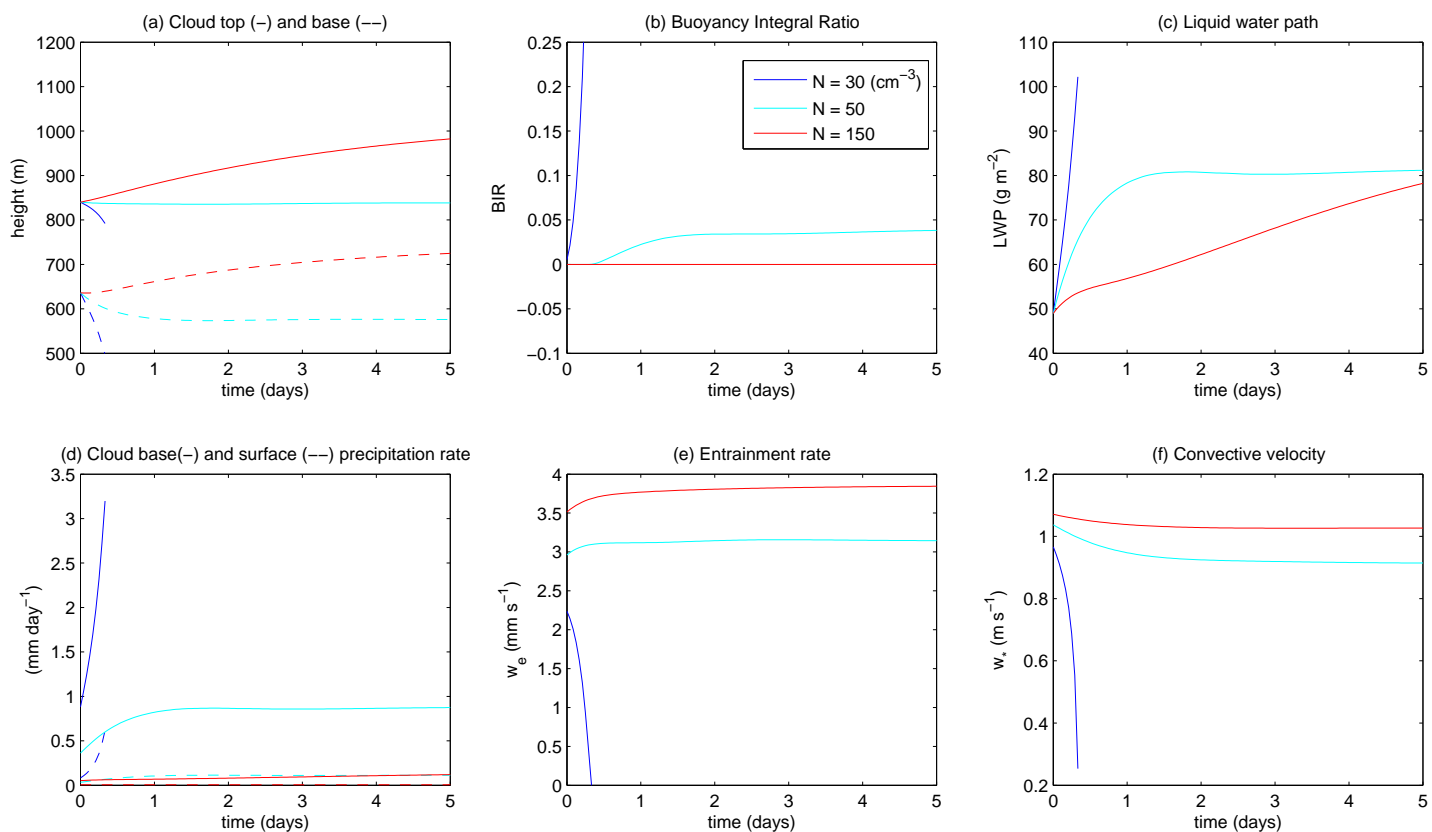

Fig. 3. MLM time series with $N=30,50$ and $150 \mathrm{~cm}^{-3}$, using standard entrainment closure: (a) cloud top (solid) and base(dashed), (b) BIR, (c) LWP, (d) cloud base (solid) and surface (dashed) precipitation rate, (e) $w_{e}$, and (f) convective velocity. Simulations are terminated if the entrainment rate drops to zero; this happens immediately for the $N=10 \mathrm{~cm}^{-3}$ case so it is not shown.

For N30, the drizzle evaporation feedback on entrainment is even stronger. As shown by the dark blue curves in Fig. 3, this leads to a runaway decrease of convective velocity (vertically integrated buoyancy flux) and entrainment accompanied by a corresponding increase of drizzle and LWP. Entrainment drops to zero after $8 \mathrm{~h}$ because convective velocity (which is the MLM predictor of boundary-layer turbulence) drops to zero. Without turbulence, the boundary layer will not remain well-mixed, so the MLM is no longer applicable. Hence no MLM results are plotted after this time. Well before this, at about $5 \mathrm{~h}$, the BIR has climbed above 0.2 , indicating significant negative subcloud buoyancy fluxes that imply that the mixed layer assumption is no longer self-consistent.

For N10, the MLM run on the initial state produces $6 \mathrm{~mm} \mathrm{~d}^{-1}$ of cloud base drizzle. The resulting subcloud cooling completely stabilizes the mixed layer, preventing convection and entrainment and rendering the MLM inconsistent from the start. Hence no N10 results are plotted on Fig. 3.

In summary, the sign of the response (an increase in LWP for a decrease in $N$ ) is the same for the MLM as for the wellmixed regime of the LES. However, the MLM simulations predict higher LWP and stronger feedbacks between cloud thickness, drizzle and droplet concentration than do the LES simulations.

\subsection{Retuning the entrainment closure to the LES}

We have suggested that the discrepancies between the MLM and LES results may arise from the LES entraining too effi- ciently compared to the observations underlying the MLM entrainment closure. For dry convective boundary layers, LES, observations, and our entrainment closure all agree that the entrainment efficiency $A \approx a_{1}=0.2$ (Stull, 1976). The choice $a_{\text {sed }}=9$ was based on prior simulations of the original GCSS DYCOMS RF01 case with our LES (Bretherton et al., 2007). In this section, we will retune the remaining nondimensional parameter in our entrainment closure, the evaporative enhancement $a_{2}$, to fit our LES results. We then test if this produces better agreement between the MLM and LES.

We start by computing the entrainment efficiency $A_{\text {LES }}$ for each hour of the LES runs using Eq. (7). Our MLM entrainment closure predicts that

$a_{2} \exp \left(-a_{\mathrm{sed}} w_{\mathrm{sed}} / w_{*}\right)=\frac{A_{\mathrm{LES}} / a_{1}-1}{\chi_{*}\left(1-\Delta b_{s} / \Delta b\right)}$.

The left hand side, the sedimentation-modified evaporative enhancement factor, isolates $a_{2}$. The sedimentation ratio $w_{\text {sed }} / w_{*}$ on the left hand side is computed from the LES output based on LWP and $N$ using Eq. (5), exactly as in the MLM. From the 2-2.25 day LES profiles shown in Fig. 2, we estimate that $w_{\text {sed }} / w_{*}=0.012,0.024,0.035$ and 0.067 for $\mathrm{N} 150, \mathrm{~N} 50, \mathrm{~N} 30$ and 10. All terms on the right-hand side can also be computed from LES output. The saturation mixing fraction $\chi_{*}$ is computed as in the MLM by using the hourlymean LWP to infer an adiabatic cloud-top liquid water content and combining this with the inversion jumps in $q_{t}$ and $s_{l}$. 


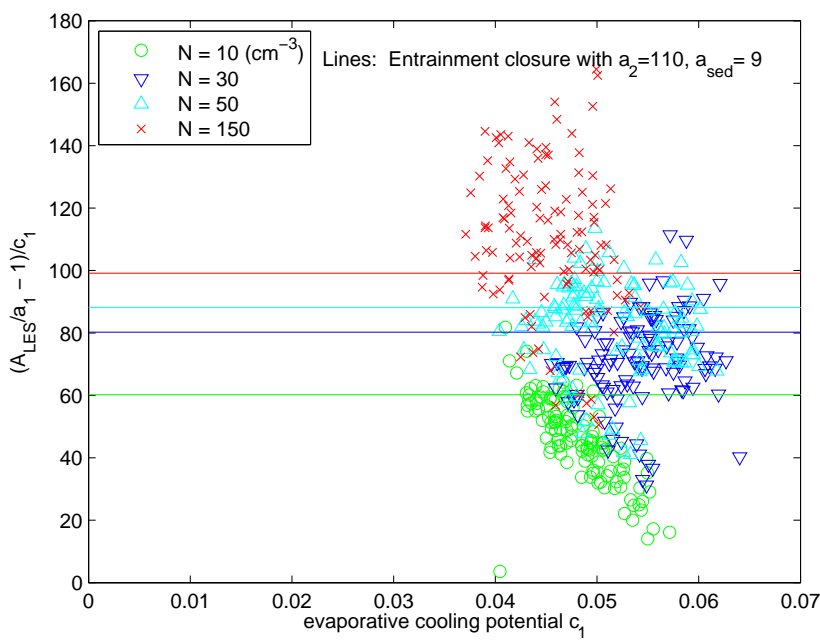

Fig. 4. Scatterplot of right hand side of Eq. (8) vs. evaporative cooling potential $c_{1}$ based on hourly-averaged LES statistics from the four simulations (symbols), which we use to tune evaporative enhancement $a_{2}$ to match the LES. Lines show predictions of our entrainment closure with $a_{2}=110, a_{\text {sed }}=9$.

Figure 4 shows a scatterplot of computed hourly averages of the right hand side vs. the relative evaporative cooling potential $c_{1}=\chi_{*}\left(1-\Delta b_{s} / \Delta b\right)$. As anticipated in the entrainment closure, the results segregate by droplet concentration, and for the well-mixed cases (all but N10), they do not show systematic residual dependence on $c_{1}$. The horizontal lines show the left hand side with $a_{2}=110, a_{\text {sed }}=9$, and the values of the sedimentation ratio $w_{\text {sed }} / w_{*}$ given above; this is a reasonable fit to the three well-mixed simulations, though less good for the N10 case. It is reassuring (though not surprising) that the choice $a_{\text {sed }}=9$ made by Bretherton et al. (2007) based on the same LES (using a different set of sensitivity studies based on the unmodified GCSS DYCOMS RF01 case) also fits the sensitivity of our simulations to sedimentation efficiency. The vertical scatter about these lines is due to uncertainty in estimating parameters and hourly entrainment rate variability unexplained by our entrainment closure. Note that the LES-tuned $a_{2}$, although it works across our simulations, cannot be expected to be a universal constant. It depends on the numerical formulation of the LES, its grid spacing near the cloud top and possibly the thermodynamic jumps at the inversion (insofar as they affect the subgrid and numerical diffusion). In the next section, we rerun the MLM with $a_{2}$ increased to 110 to see if it better matches the LES results.

\subsection{MLM simulations with $a_{2}=110$}

Figure 5 shows time series from the MLM run with $a_{2}=110$ for the three cases N30, N50 and N150 which the LES predicts are well mixed. In all cases, the evolution of the LWP over the first two days is much closer to the LES than in the case $a_{2}=25$. This shows the control of entrainment efficiency on cloud thickness. Nevertheless, the N30 simulation has much more cloud base drizzle and less entrainment than their LES analogues. This initiates a drizzle-induced boundary layer collapse by the same entrainment-mediated mechanism we saw in the $a_{2}=25$ MLM simulation.

The modified entrainment closure greatly improves the agreement of MLM and LES as long as the MLM predicts less than $0.15 \mathrm{~mm} \mathrm{day}^{-1}$ of cloud base drizzle. Once the MLM-simulated cloud starts to drizzle even a little, the MLM starts to diverge from the LES. This difference can be traced to the difference between LES and MLM in simulated drizzle for a given amount of LWP. In the MLM, the cloud base precipitation flux in units of $\mathrm{mm} \mathrm{d}^{-1}$ is parameterized as $0.37(\mathrm{LWP} / N)^{1.75}$ following Comstock et al. (2004) (here and below, LWP/N is in units of $\mathrm{g} \mathrm{m}^{-2} \mathrm{~cm}^{3}$ ). Figure 6 shows the LES-derived hourly-averaged cloud base precipitation $P_{\mathrm{cb}}$ vs. LWP/N across the four simulations, plus some additional simulations of the much drizzlier DYCOMS RF02 case, along with a solid line showing the power-law fit

$P_{\mathrm{cb}}^{\mathrm{LES}}=0.023(\mathrm{LWP} / N)^{3.25}$.

This is qualitatively similar to a fit $P_{\mathrm{cb}} \propto \mathrm{LWP}^{3.7} N^{-2.3} \mathrm{ob}-$ tained by Geoffroy et al. (2008) from an LES with a related bulk microphysical scheme. Compared with the MLM drizzle parameterization (the dashed line on the figure), our LES has a more threshold-like behavior, with very little cloud base drizzle at low LWP/N. This makes the MLM more susceptible than the LES to reduction of entrainment by drizzle at moderate LWP.

Because area-mean cloud base drizzle becomes very hard to measure when it is weak, the observational fit used in the MLM may not be trustworthy at drizzle rates much less than $1 \mathrm{~mm} \mathrm{~d}^{-1}$. Hence the discrepancy between the MLM and LES drizzle predictions for low drizzle rates, while large, may lie within observational uncertainty. In the heavilydrizzling stratocumulus intercomparison of Ackerman et al. (2009), the SAM LES predicted the cloud-base drizzle rate within observational uncertainty, performing at least as well as most other participating LESs.

\subsection{MLM simulations with $a_{2}=110$ and LES-tuned cloud base drizzle}

If we use Eq. (9) in place of the default MLM cloud-base drizzle parameterization, in addition to using the LES-tuned entrainment parameter $a_{2}=110$, the resulting MLM simulations of LWP and cloud-base drizzle for N30, N50 and N150 (Fig. 7) match the LES results more closely. Now the MLM, like the LES, does not exhibit drizzle-induced boundary layer collapse even for N30, remaining self-consistently coupled with $\mathrm{BIR}<0.05$ over the 5 -day simulation period. The sensitivities of LWP and $w_{*}$ to $N$ are comparable in the two models. Both have a 20-30\% increase in LWP and a 5-10\% increase in $w_{*}$ as $N$ decreases from $150 \mathrm{~cm}^{-3}$ to $30 \mathrm{~cm}^{-3}$. 

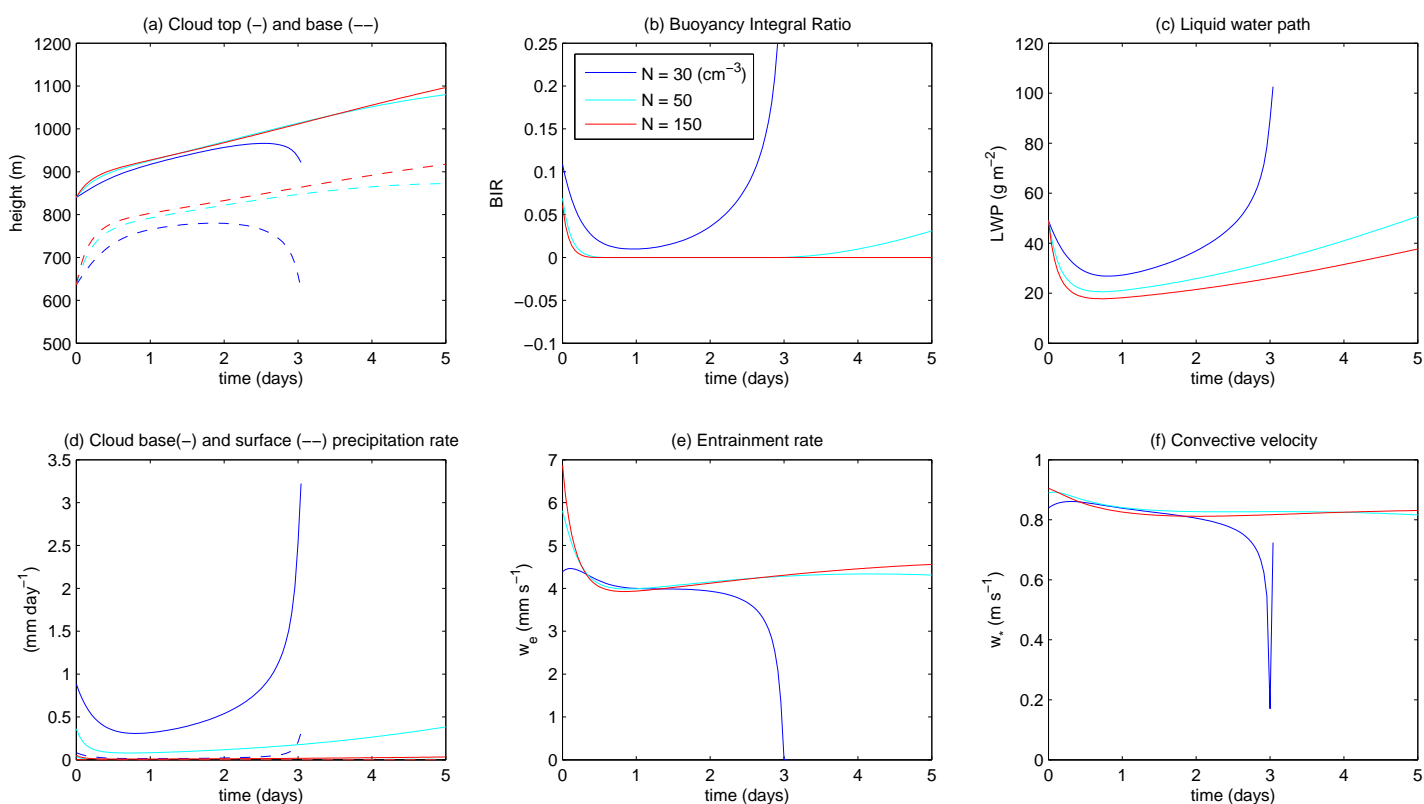

Fig. 5. As in Fig. 3, but using $a_{2}=110$ in the MLM entrainment closure.

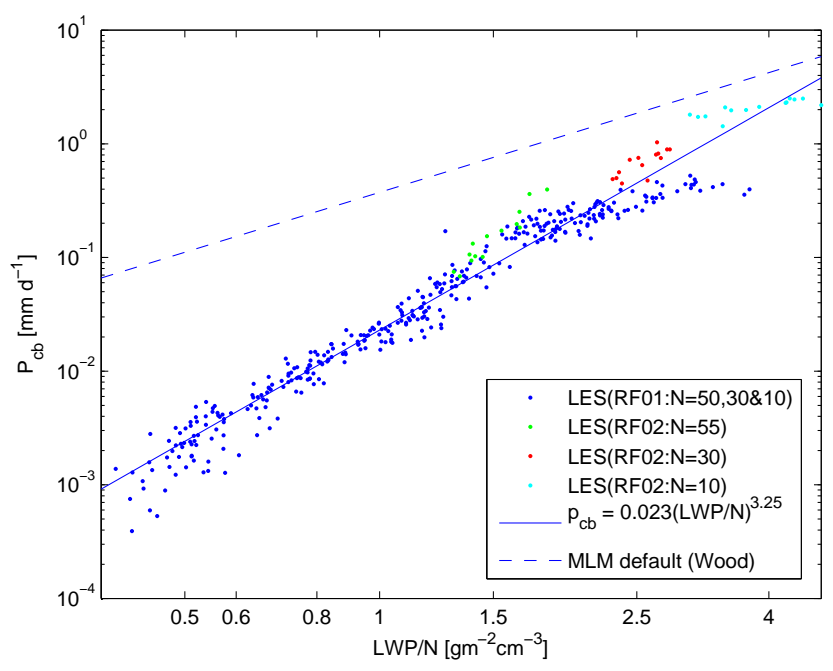

Fig. 6. LES cloud base precipitation rate vs. LWP/N. Dots indicate hourly averages from the 4 LES cases; line indicates the power law fit of Eq. (9), and the dashed line shows the default MLM parameterization.

The LES-tuned MLM also reproduces key LES vertical profiles fairly well, as shown in Fig. 2 for N150. This is particularly noteworthy for the liquid water profile (Fig. 2c) and buoyancy flux profile (Fig. 2e), which are quite sensitive to both vertical and horizontal inhomogeneity in boundarylayer air properties. The LES buoyancy flux profile is rounded off within the cloud layer compared to the MLM because the MLM assumes $100 \%$ cloud cover above the mean cloud base and no cloud below this level, while the LES cloud fraction transitions more gradually due to the range of saturation levels between the updrafts and downdraft. Nevertheless, the MLM captures the overall structure of the LES buoyancy flux profile quite well.

The MLM does not show as much sensitivity of inversion height to $N$ as does the LES. The separation of LES inversion heights for different $N$ occurs between 1.5 and 2 days, when the cloud in the N150 simulation becomes thin and the cloud fraction decreases to $80 \%$. This reduces the boundary layer radiative driving for turbulence, the entrainment rate, and ultimately the inversion height compared to the N30 simulation in which cloud cover remains $85 \%$ or higher throughout. A comparable sensitivity of radiative driving to cloud fraction cannot occur in the MLM, where by construction the cloud fraction remains $100 \%$ even as the cloud becomes very thin.

For N10, the MLM initially drizzles enough to predict immediate decoupling (initial $\mathrm{BIR}=0.25$ ); this is consistent with the decoupled boundary layer simulated by the LES.

The MLM drizzle parameterization is an empirical fit encapsulating observed relationships, and the LES microphysics can be significantly biased compared to observations, as other intercomparisons have shown (Ackerman et al., 2009). Thus, as with entrainment, the LES predictions are arguably less plausible than the MLM as long as the boundary layer remains well mixed.

\section{Discussion}

Our results can be used to estimate the relative importance of the second and first indirect effects in our simulations, which Wood (2007) quantified in terms of an indirect effect 

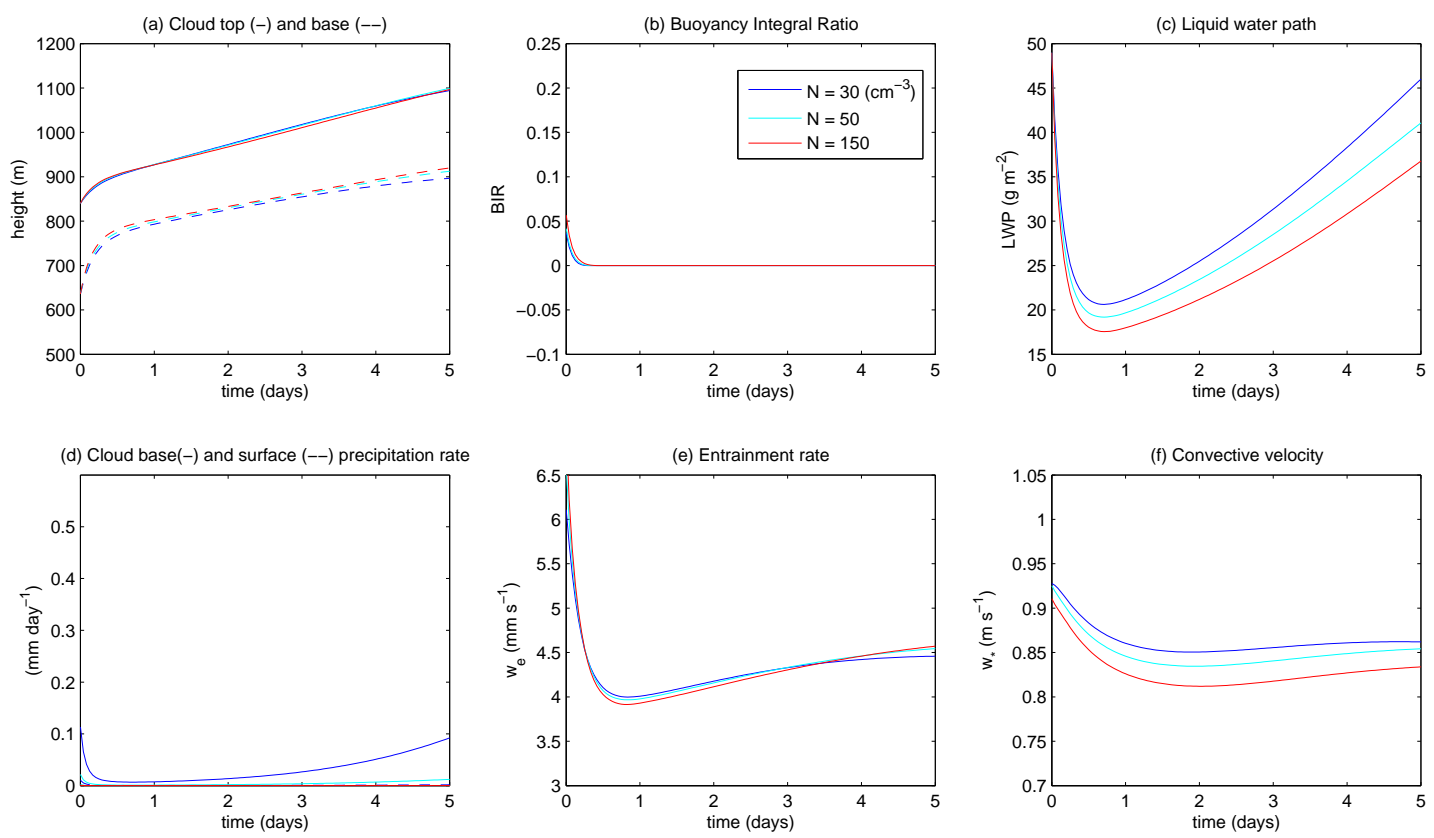

Fig. 7. MLM time series as in Fig. 3 but with $a_{2}=110$ and LES-tuned cloud base drizzle.

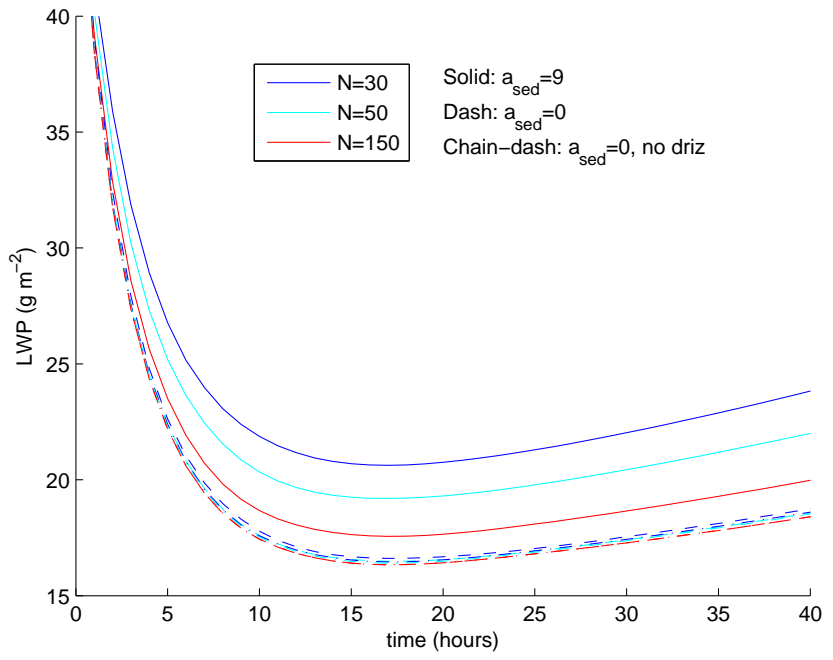

Fig. 8. 40-h time series of MLM-simulated LWP for $N=150,50$ and $30 \mathrm{~cm}^{-3}$ with $a_{\text {sed }}=9$ (solid) and $a_{\text {sed }}=0$ (dashed). LES-tuned cloud-base drizzle and $a_{2}=110$ are used. The chain-dash curves, almost indistinguishable from the dashed curves, show the negligible effect of suppressing drizzle on the $a_{\text {sed }}=0$ simulations.

ratio. For well-mixed stratocumulus-capped boundary layers, Wood's analysis implies that

$R_{I E}=2.5 \partial \ln \mathrm{LWP} / \partial \ln N$.

This formula is strictly applicable to an MLM, in which the cloud is horizontally homogeneous, but should also be a reasonable estimate for the LES in a well-mixed, nearly fully cloud-covered regime. Wood focused on MLM simulations with significant drizzle and found that in that case, $R_{I E}$ is typically positive (i.e. second indirect effect reinforcing the first) and can exceed two. He also noted that $R_{I E}$ is particularly sensitive to the simulated cloud base, and becomes negative for his parameter choices when the simulated cloud base exceeds $400 \mathrm{~m}$ (as in our simulations).

Our LES and MLM simulations of nearly nondrizzling stratocumulus have negative $R_{I E}$, since LWP decreases as $N$ increases. The MLM $R_{I E}$ depends on whether its parameters are tuned to observations or to LES. Comparing N30 to $\mathrm{N} 150(\delta \ln N=1.6)$, the average $\delta \ln L W P \approx-0.2$ for the LEStuned MLM and -0.3 for the LES over the period from $16 \mathrm{~h}$ to 5 days, after the simulations have gone beyond their initialization transients. This implies an $R_{I E}$ of -0.3 (LES-tuned MLM) or -0.5 (LES) for these simulations, i.e. a significant partial cancellation of the first indirect (Twomey) effect by the LWP changes associated with sedimentation feedbacks. This encouraging level of agreement supports the use of an MLM to estimate $R_{I E}$ in this regime, as long as sedimentation feedback is included in the entrainment closure, the MLM-predicted buoyancy flux remains positive throughout the subcloud layer, and the LWP exceeds $20 \mathrm{~g} \mathrm{~m}^{-2}$ (a rough threshold for cloud cover to remain close to $100 \%$, at least for our modest LES domain size). We caution that the LES $R_{I E}$ is sensitive to the assumed physics and grid reolution in the entrainment zone, including effects we have neglected such as finite evaporation and grid-box mixing timescales. The same is true of the MLM, since its sedimentation feedback is tuned to the LES. 
For the cases simulated here, the empirically-tuned MLM would have a more negative $R_{I E}$ (at least before the BIR criterion predicts decoupling) than the LES-tuned MLM, due to the higher propensity of the observationally-tuned MLM to drizzle and hence a stronger microphysical feedback on entrainment. This more negative $R_{I E}$ may actually be more realistic than the LES results, because of the LES entrainment and microphysical biases we have noted. That is, we should be wary of current LES predictions of $R_{I E}$ in stratocumulus regimes.

Our MLM results contrast with those of S2009, who found that their MLM tended to produce a thicker cloud if droplet concentration is higher. We believe that the difference lies mainly in their choice of entrainment closure. Much of S2009's paper is an excellent tutorial of how to evaluate whether a MLM is an adequate representation of an LES of a stratocumulus-capped boundary layer. S2009, like us, tried to carefully select an entrainment closure that matched their LES. They selected from a set of entrainment closures that have been developed for use in real stratocumulus cloudtopped mixed layer. They selected the original NichollsTurton closure (with no sedimentation feedback and $a_{2}=60$ ) because it gave a prediction of $w_{e}$ that had the smallest bias averaged over all of their cases compared to the LES, while predicting the temporal and case-to-case variation of entrainment rate comparably well to other closures that they considered (see their Table 4). Other closures, including a variation of the one used in this paper, better predicted the case-to-case variation of entrainment rate but were rejected because they produced a mean entrainment rate bias compared to the LES.

This is an appealing approach, but it makes the tacit assumption that their LES gives an unbiased representation of a stratocumulus-capped boundary layer and hence should satisfy the same entrainment closure as derived from observations. As we showed in Sect. 3.3, our LES entrains too efficiently and clearly does not satisfy this assumption. We must use $a_{2}=110$ in our version of the Nicholls-Turton closure to match the LES results. Recent observational studies that have evaluated the Nicholls-Turton closure adopted by S2009 using modern observations have concluded that one should take $a_{2}=15-30$ (Stevens et al., 2003; Caldwell et al., 2005). This is much less than the $a_{2}=60$ originally suggested by NT86 on the basis of a very small set of aircraft observations of stratocumulus, some of which were not even in mixed layers. Viewed from this perspective, S2009's LES also appears to entrain too efficiently compared to observations, requiring an $a_{2}=60$ that is more than twice as large as the observational consensus (though not as extreme as the $a_{2}=110$ we require to fit the SAM LES). Overefficient entrainment is an almost universal feature of LES models at current resolution, as shown by their underestimation of LWP in the GCSS RF01 intercomparison (Stevens et al., 2005b).

Given this bias in LES entrainment, it is no surprise that observationally-based entrainment closures tend to underestimate the LES entrainment rate, as seen in the Bretherton et al. (2007) and Lilly and Stevens (2008) closures of S2009's Table 4. This does not mean those closures should be rejected. Instead, if our goal is to interpret a LES using a mixed layer model, we may want to use different, retuned versions of those closures that match the LES, as we have done in this paper. Unfortunately, some aspects of entrainment closure, such as the sensitivity of entrainment efficiency to droplet sedimentation, are very hard to constrain using current observations (perhaps ship tracks could be used for this purpose), rendering LES a crucial tool for understanding such effects. The sedimentation correction to entrainment is the primary mechanism for a second indirect effect for the nearly nondrizzling stratocumulus layers in our LES and MLM simulations. Figure 8 explores how removing the sedimentation correction to entrainment (i.e. taking $a_{\text {sed }}=0$ in our MLM) affects the response of the MLM to a droplet concentration change. Without the sedimentation correction, the MLM LWP has almost no sensitivity to droplet concentration at high $N$ or to the suppression of drizzle.

We concur with S2009 that some other effects of droplet sedimentation (such as its effect on the vertical structure of the buoyancy flux profile) are captured in an MLM without a sedimentation correction to the entrainment closure, but these effects clearly have a negligible impact on the simulated LWP in the simulations shown in Fig. 8.

The MLM of S2009 also did not include the effect on $w_{*}$ of drizzle that evaporates below cloud base, another key effect that decreases turbulence and entrainment in our MLM (see Fig. 2 of Bretherton et al., 2007) and in LES (e.g. Stevens et al., 1998).

S2009 found that their MLM produces a higher LWP when $N$ is larger. According to their Fig. 8, this effect builds during the night, maximizing at dawn, then goes away during the day. Interestingly, their LES has a similar diurnal change of the LWP difference between low and high $N$ as in the MLM, even though the daily-mean LWP difference is quite different than for the MLM. This result deserves further study. It may arise from dependence of radiative heating rates on $N$, an effect included in their study and not in ours.

\section{Conclusions}

We find that for a well-mixed stratocumulus-topped boundary layer, an LES and a suitably configured mixed layer model predict a similar decrease of cloud liquid water path as droplet concentration increases. In both models, this effect produces cancellation of 30-50\% of the Twomey effect for our case.

However, LES advection schemes and microphysical parameterizations can significantly bias the entrainment rate and precipitation profile compared to observational best guesses. To obtain results comparable to the LES, the entrainment and drizzle parameterizations of the MLM must be carefully tuned to the LES. This causes the MLM-simulated 
cloud layer to be thinner and drizzle less compared to a simulation with the observationally-based default MLM parameterizations. In some cases, an MLM simulation with the default parameterizations will produce a drizzling boundary layer which collapses due to drizzle-entrainment feedback, while the LES-tuned MLM simulation produces a thin, nondrizzling cloud evolving toward a steady state. This suggests that potential LES biases must be carefully considered when trying to make a quantitative inference of the second indirect effect from an LES of a stratocumulus-topped boundary layer.

On the other hand, several points must also be kept in mind when inferring the second indirect effect from MLM simulations. First, the MLM is only valid when the boundary layer remains well mixed, as diagnosed using a BIR criterion. Drizzle and insolation both promote decoupling, and the MLM should not be trusted when BIR exceeds 0.1. Second, the MLM entrainment closure plays a crucial role in diagnosing when decoupling is likely as well as in the representation of entrainment-sedimentation feedback, and there is no consensus on the choice of closure. Third, if the simulated cloud gets too thin, then the MLM assumption of $100 \%$ cloud cover is no longer reasonable. That is, the transition from a non-cloudy to a cloudy boundary layer is smoother in reality than an MLM allows.

These considerations underline the need for LES with advection schemes that accurately represent entrainment and microphysical schemes that can accurately represent observed relationships between cloud thickness, cloud morphology, and precipitation rate. Recent GCSS intercomparisons (Stevens et al., 2005b; Ackerman et al., 2009) suggest a few LES may be close to achieving these goals. Careful observationally-grounded comparisons of such LES with simpler parameterization approaches such as MLMs and single-column versions of climate models will continue to be needed to underpin our understanding of aerosol indirect effects.

Acknowledgements. We would like to acknowledge support from NASA grant NNX09AH73G and NSF grant ATM-0745702, helpful discussions with Rob Wood and Irina Sandu, and comments from two anonymous referees.

Edited by: T. Garrett

\section{References}

Ackerman, A., Toon, O. B., Stevens, D. E., and Coakley Jr, J. A.: Enhancement of cloud cover and suppression of nocturnal drizzle in stratocumulus polluted by haze, Geophys. Res. Lett., 30(7), 1381, doi:10.1029/2002GL016634, 2003.

Ackerman, A., Kirkpatrick, M. P., Stevens, D. E., and Toon, O. B.: The impact of humidity above stratiform clouds on indirect aerosol climate forcing, Nature, 432, 1014-1017, 2004.
Ackerman, A. S., vanZanten, M. C., Stevens, B., and coauthors: Large-eddy simulations of a drizzling, stratocumulus-topped marine boundary layer, Mon. Weather Rev., 137, 1083-1110, 2009.

Baker, M. B. and Charlson, R.: Bistability of CCN concentrations and thermodynamics in the cloud-topped boundary layer, Nature, 345, 142-145, 1990.

Bretherton, C. S. and Wyant, M. C.: Moisture transport, lower tropospheric stability and decoupling of cloud-topped boundary layers, J. Atmos. Sci., 54, 148-167, 1997.

Bretherton, C. S., Uttal, T., Fairall, C. W., Yuter, S., Weller, R., Baumgardner, D., Comstock, K., and Wood, R.: The EPIC 2001 stratocumulus study, B. Am. Meteorol. Soc., 85, 967-977, 2004.

Bretherton, C. S., Blossey, P. N., and Uchida, J.: Cloud droplet sedimentation, entrainment efficiency, and subtropical stratocumulus albedo, Geophys. Res. Lett., 34, L03813, doi:10.1029/2006GL027648, 2007.

Caldwell, P., Bretherton, C. S., and Wood, R.: Mixed-layer budget analysis of the diurnal cycle of entrainment in Southeast Pacific stratocumulus, J. Atmos. Sci., 62, 3775-3791, 2005.

Caldwell, P. and Bretherton, C. S.: Response of a subtropical stratocumulus-capped mixed layer to climate and aerosol changes, J. Climate, 22, 20-38, 2009.

Coakley Jr, J. A. and Walsh, C. D.: Limits to the aerosol indirect radiative effect derived from observations of ship tracks, J. Atmos. Sci., 59, 668-680, 2002.

Comstock, K. K., Wood, R., Yuter, S. E., and Bretherton, C. S.: Reflectivity and rain rate in and below drizzling stratocumulus, Q. J. Roy. Meteorol. Soc., 130, 2891-2918, 2004.

Comstock, K. K.,Bretherton, C. S., and Yuter, S. E.: Mesoscale variability and drizzle in southeast Pacific stratocumulus, J. Atmos. Sci., 62, 3792-3807, 2005.

Gasso, S.: Satellite observations of the impact of weak volcanic activity on marine clouds, J. Geophys. Res., 113, D14S19, doi:10.1029/2007JD009106, 2008.

Geoffroy, O., Brenguier, J.-L., and Sandu, I.: Relationship between drizzle rate, liquid water path and droplet concentration at the scale of a stratocumulus cloud system, Atmos. Chem. Phys., 8, 4641-4654, 2008, http://www.atmos-chem-phys.net/8/4641/2008/.

Grenier, H. and Bretherton, C. S.: A moist PBL parameterization for large-scale models and its application to subtropical cloudtopped marine boundary layers. Mon. Weather Rev., 129, 357377, 2001.

Hill, A. A., Feingold, G., and Jiang, H.: The influence of entrainment and mixing assumption on aerosol-cloud interactions in marine stratocumulus, J. Atmos. Sci., 66, 1450-1464, 2009.

Khairoutdinov, M. F. and Kogan, Y. L.: A new cloud physics parameterization in a large-eddy simulation model of marine stratocumulus, Mon. Weather Rev., 128, 229-243, 2000.

Khairoutdinov, M. F. and Randall, D. A.: Cloud resolving modeling of the ARM Summer 1997 IOP: Model formulation, results, uncertainties, and sensitivities, J. Atmos. Sci., 60, 607-625, 2003.

Larson, V. E. and Kotenberg, K. E.: An analytic longwave radiation formula for liquid layer clouds, Mon. Weather Rev., 135, 688699, 2007.

Lilly, D. K. and Stevens, B.: Validation of a mixed-layer closure. Part I: Theoretical tests, Q. J. Roy. Meteorol. Soc., 134, 47-55, 2008.

Lu, M.-L., and Seinfeld, J. H.: Study of the aerosol indirect effect by 
large-eddy simulation of marine stratocumulus, J. Atmos. Sci., 62, 3909-3932, 2005.

Nicholls, S. and Turton, J. D.: An observational study of the structure of stratiform cloud layers: Part II: Entrainment, Q. J. Roy. Meteorol. Soc., 112, 461-480, 1986.

Petters, M. D., Snider, J. R., Stevens, B., Vali, G., Faloona, I., and Russell, L. M.: Accumulation mode aerosol, pockets of open cells, and particle nucleation in the remote subtropical Pacific marine boundary layer, J. Geophys. Res., 111, D02206, doi:10.1029/2004JD005694, 2006.

Pincus, R. and Baker, M. B.: Effect of precipitation on the albedo susceptibility of marine boundary layer clouds, Nature, 372, 250-252, 1994.

Randall, D. A.: Conditional instability of the first kind upsidedown, J. Atmos. Sci., 37, 125-130, 1980.

Sandu, I., Brenguier, J.-L., Thouron, O., and Stevens, B.: How important is the vertical structure for the representation of aerosol impacts on the diurnal cycle of marine stratocumulus?, Atmos. Chem. Phys., 9, 4039-4052, 2009, http://www.atmos-chem-phys.net/9/4039/2009/.

Savic-Jovcic, V. and Stevens, B.: The structure and mesoscale organization of precipitating stratocumulus, J. Atmos. Sci., 65, 15871605, 2008.

Stevens, B.: Cloud transitions and decoupling in shear-free stratocumulus-topped boundary layers, Geophys. Res. Lett., 27, 2557-2560, 2000.

Stevens, B., Cotton, W. R., Feingold, G., and Moeng, C.H.: Large-eddy simulations of strongly precipitating, shallow, stratocumulus-topped boundary layers, J. Atmos. Sci., 55, 36163638, 1998.

Stevens, B., Lenschow, D. H., Faloona, I., and coauthors: On entrainment rates in nocturnal marine stratocumulus, Q. J. Roy. Meteorol. Soc., 129, 3469-3493, 2003.
Stevens, B., Vali, G., Comstock, K., Wood, R., van Zanten, M. C., Austin, P. H., Bretherton, C. S., and Lenschow, D. H.: Pockets of open cells (POCs) and drizzle in marine stratocumulus, B. Am. Meteorol. Soc., 86, 51-57, 2005a.

Stevens, B., Moeng,, C.-H., Ackerman, A. S., and coauthors: Evaluation of large-eddy simulations via observations of nocturnal marine stratocumulus, Mon. Weather Rev., 133, 1443-1462, 2005b.

Stull, R. B.: The energetics of entrainment across a density interface, J. Atmos. Sci., 33, 1260-1267, 1976.

Smolarkiewicz, P. K., and W. W. Grabowski: The multidimensional positive definite advection transport algorithm: Non-oscillatory option, J. Comput. Phys. 86, 355-375, 1990.

Wood, R.: Cancellation of aerosol indirect effects in marine stratocumulus through cloud thinning, J. Atmos. Sci., 64, 26572669, 2007.

Wood, R., Comstock, K. K., Bretherton, C. S., Cornish, C., Tomlinson, J., Collins, D. R., and Fairall, C.: Open cellular structure in marine stratocumulus cloud sheets, J. Geophys. Res., 113 D12207, doi:10.1029/2007JD009371, 2008.

Xue, H., Feingold, G., and Stevens, B.: The role of precipitating cells in organizing shallow convection, J. Atmos. Sci., 65, 392406, 2008.

Wyant, M. Bretherton, C. S., Chlond, A., and coauthors: A single column model intercomparison of a heavily drizzling stratocumulus topped boundary layer, J. Geophys. Res., 112, D24204, doi:10.1029/2007JD008536, 2007.

Zhu, P., Bretherton, C. S., Koehler, M., and coauthors: Intercomparison and interpretation of single column model simulations of a nocturnal stratocumulus topped marine boundary layer, Mon. Weather Rev., 133, 2741-2758, 2005. 